\title{
Prevalence of Burnout Syndrome in Female Elementary School Teachers of Karachi, Pakistan
}

\author{
Kiran Khan, ${ }^{1}$ Sadia Sundus, ${ }^{2}$ Ghazala Nasim Pasha, ${ }^{3}$ Sarwat Fatmee, ${ }^{4}$ Ata ur Rehman, ${ }^{5}$ Wazir Ahmed ${ }^{6}$
}

\begin{abstract}
Background: Female teachers like other professionals, also face stress and exhaustion due to their jobs, which may lead to burnout.

Objective: To investigate the various degrees of burnout and its association with demographic factors on female teachers of private and public sector elementary schools, Karachi Pakistan.

Methodology: This cross-sectional study was carried out on 800 female teachers in Karachi Pakistan, from January to May 2017. MBI-HSS was used as an instrument for this extensive study, data was stored and analyzed on IBM-SPSS version 22.0. Pearson chi-square was done to see the association of demographics with burnout, one-way ANOVA to compare scores of MBI with marital status and zone, Tukey's test for multiple comparisons between the groups, and Cronbach's alpha to evaluate the measure of internal consistency.

Results: The results revealed that among the female elementary school teachers, a total of $96.9 \%$ reported burnout (13\% mild, $58 \%$ moderate, $19.6 \%$ severe, $6.3 \%$ extreme) and $3.1 \%$ had no burnout. Burnout varies with marital status (highest in married participants) and zone (highest in east zone) (p values $<0.05$ ) only. One-way ANOVA demonstrate that the separated women had higher mean scores on MBI scales compared to married \& unmarried women (p-value $=0.48)$, furthermore, East zone respondents have higher MBI scores as compared to all other regions. Tukey's test showed that East zone gave significant mean differences for south and west zone (P-value $<0.05)$. Cronbach's alpha was found to be $(0.384)$ for the overall scale confirming the reliability of subscales and MBI.

Conclusion: Our results show a high prevalence of burnout and the positive association of Burnout with marital status and zones.
\end{abstract}

Keywords: Burnout, Female, Teachers, Demographic

Article Citation:Khan k, Sundus S, Pasha GN, Fatmee S, Rehman A, Ahmed W. Prevalence of Burnout Syndrome in Female Elementary School Teachers of Karachi, Pakistan. JSZMC 2021;12(4):10-15. DOI: https://doi.org/10.47883/jszmc.v11i04.167

This Open Access article in Journal of Sheikh Zayed Medical College is licensed under a Creative Commons Attribution-NonCommercial 4.0 International License.

\section{Introduction}

There are many phases in people's lives when they face stress and exhaustion due to their jobs. The escalation of these feelings over time may lead a person towards burnout. ${ }^{1,2}$ These days teaching students is becoming more challenging because of their lack of interest in school activities, disrespectful and bored attitude. They are least interested in attending school regularly, due to coaching services. ${ }^{3,4}$ It has been observed that Jobs with relational contact with others tend to have increased levels of burnout. ${ }^{5,6}$ Many past researches have recognized teaching as an extremely challenging occupation, linked with high intensities of burnout.
A number of studies have been conducted and multiple reasons were highlighted that lead a teacher towards this deterioration and were found to be multifactorial, such as; student factors, which include causes such as disciplinary problems, students dislike for studies, lack of interest, and low output. Out of the many adverse effects of teacher burnout, the undesirable influences on the students are the biggest problems when it comes down to schools. ${ }^{8,9}$ There are also job factors influencing female school teachers' health and so the burnout including low salaries, lack of authority, low appreciation, and lack of motivation and acknowledgment. ${ }^{9,10}$ The school factors include pressure from parents and organization, tense

1.Department of Community Medicine, Iqra Medical \& Dental College, Karachi.

2.Department of Anatomy, Iqra Medical \& Dental College, Karachi.

3.Department of Community Medicine, United Medical \& Dental College, Karachi.

4.Department of Anatomy, Fatima Jinnah Dental College, Karachi.

5.Department of Pharmacology, Hamdard College of Medicine \& Dentistry, Karachi.

6.Department of Anatomy, Makran Medical College, Turbat, Pakistan.

Correspondence: Dr. Sadia Sundus, Associate Professor, Department of Anatomy, Iqra Medical \& Dental College, Karachi. Email: usadsun_dr@yahoo.com Received: $30-09-2020$ Revised: $28-10-2021$ 
interpersonal relationships, lack of support, ineffective educational reforms, and poor school environment. The personal factors include excessively high expectations, inadequate capabilities, and requirement of occupational development correlated distress is an additional source of stress for teachers. This happens when there is a dissimilarity between what a teacher is prepared for against the environment which they encounter at the time of teaching. ${ }^{10,11}$ The terms burnout and stress are substitutable to each other, this is culpability in accepting the actual nature of burnout. Burnout occurs in a substantial interval of period. However outstanding teachers try to cope with stress by actively trying to solve the problem.12 However, failure to withstand against such chronic stress, cause "burnout" among teachers. ${ }^{13}$

Burnout can be conceptualized as three distinct dimensions. Emotional Exhaustion denotes feelings of being emotionally overreached and fatigue of one's emotional means. ${ }^{14,15}$ Depersonalization denotes loss of idealism, insensitive, or excessively isolated behavior with other people. ${ }^{16}$ Reduced Personal Accomplishment, is a self-evaluation component and it represents a downfall in one's feelings of achievement and fitness at work. A study conducted evaluated the distinct factors leading to burnout in private and public school teachers. It was revealed that the working conditions of the job itself caused emotional exhaustion in the public sector schools, whereas the immediate supervisor and overload caused emotional exhaustion among the private sector. This approach to study burnout sheds more light on its comprehensive mechanism. It emphasizes distinguishing features between public and private school systems in terms of burnout assessment. ${ }^{17,18}$ The objective of this study was to investigate the various degrees of burnout and its association with demographic factors on female teachers of private and public sector elementary schools, Karachi Pakistan..

\section{Methodology}

This cross-sectional study was conducted on 800 female elementary school teachers from Karachi, Pakistan in $1^{\text {st }}$ January to 30May 2017 . More than 50 schools in Karachi were surveyed from both the sectors (private and public) for the purpose of data collection. Elementary school teachers from both private and public sectors in the ratio of 1:1 were selected for the study (400 from private and public sectors each). The sample was further categorized according to 5 districts of Karachi namely north, south, east, west, and central with a number of 80 teachers from each district per sector. Female participants having at least 1 year work experience at the same school, aged between 18-65 years, and willing to participate were included for this research. Teachers on temporary contracts or ones with an experience of less than 1 year, nonteaching staff (male/female) were excluded. The participants were further categorized on the basis of marital status (married, unmarried, separated, widowed), and educational status (matriculation, graduation, and masters) respectively

The management of all the schools was informed about the research to obtain approval. Informed consent was taken from all the participants and ethical considerations and confidential protection of all individuals were observed. It was ethically approved by the department review committee of the Community Health Department, Hamdard College of Medicine and Dentistry, Hamdard University, Karachi.

The MBI questionnaire was used in it, recognized as the significant measure of burnout which was developed in the 1970s. We used the MBI-HSS version, a widely used version of MBI, to pursue the extensive study. The MBI evaluates the key features of burnout disorder, emotional exhaustion, depersonalization, and personal accomplishment. The final form of the first MBI scale, the MBI-HSS contains 22-items. The 9 items come under emotional exhaustion. The emotional exhaustion subscale assesses the feelings of being emotionally strained and exhausted by one's effort. The depersonalization subscale comprises 5 objects, which analyze a detached reaction towards the recipient of the participant. For both of these categories greater mean score relates to the greater degree of burnout being practiced. Personal accomplishment consists of 8 objects which precise the feelings of skill, self-assessment, and fruitful achievement in one's effort. Low scores of personal achievement reflect high degree burnout. Each subscale has 2 dimensions; first frequency (how often people feel this way) and second intensity (the degree or strength of these feelings) the questionnaire consists of a structured Likert scale 
reaching from 0 (never) to 6 (daily), describing the various degrees relevant to the questions being asked. Each participant can choose the numerical figure appropriate for their answer and fill it in the space provided against the questions.

A 22 items from MBI-HSS and 7 items to collect demographic details, along with an Urdu translation on the other side, a final layout of the questionnaire was prepared consisting of 28 items in total, which was distributed among the teachers. MBI takes about 20-30 minutes to fill out. It was distributed, explained in detail with complete instructions after obtaining informed consent. A pilot study among 30 teachers was previously conducted to minimize response biases and to attain the validity of the questionnaire for the respective study. It was characterized by the factors such as respondent privacy; after distributing an official recommendation letter issued from our respective university, and taking approval from the school administration, the questionnaires were distributed among the female teachers. Proper privacy was maintained so that the answers are not affected by the opinion of others. The teachers filled out their questionnaires and handed them back. Respondent confidentiality: The questionnaire was designed in a way that it demands some personal information and experiences to be shared thoroughly on open grounds, therefore the further demographic questions were entered in such a selective manner that the personal identity or confidentiality of the participant remained intact. (Name or addresses of the participant or their school were excluded). Therefore the promising confidentiality assured the proper answering. The questionnaires distributed among the teachers were collected hand to hand and some were collected later with completely filled out answers required for the analysis of burnout.

Data were collected and evaluated using IBMSPSS version 23.0, percentages and sum were recounted for Burnout, Emotional exhaustion, Depersonalization, and Personal Accomplishment. Pearson chi-square test was used to understand the connotation of burnout with age group, marital status, education, elementary school, work experience, and zone, One Way ANOVA was done to compare the mean scores of MBI across marital status and zone, and for further post-hoc investigation of significant results. Tukey test was done for multiple comparisons between groups. P-values less than 0.05 revealed significance. To evaluate the measure of internal consistency, using reliability analysis Cronbach's alpha for each factor was calculated. For this study Cronbach's alpha coefficient was found to be $(0.637)$ for Emotional exhaustion, $(0.650)$ for depersonalization, $(0.737)$ for personal accomplishment, and (0.384) for combined scale, confirming the reliability of all scales and MBI as a valid choice of instrumentation for assessing burnout respectively.

\section{Results}

The study participants had a mean age of $40 \pm 11$ (range: 18-65), mean job experience of $14.73 \pm 10$ years (range: 1-60). Results of this study revealed, that of a total of 800 surveyed participants, $96.9 \%$ were reported to have burnout, among them, 13\% were at the mild stage, $58 \%$ at moderate stage, $19.6 \%$ at the severe stage, and $6.3 \%$ at extreme levels of burnout, only $3.1 \%$ of the participants were found normal for burnout. This scale further revealed that $37.5 \%$ were at a moderate level of emotional exhaustion, $21.6 \%$ at a moderate level of depersonalization, and $22.4 \%$ at a moderate level of personal accomplishment. (Table-I)

Table-I: Burnout, Emotional exhaustion, Depersonalization, and Personal accomplishment among study subjects. $(n=800)$

\begin{tabular}{|c|c|c|c|}
\hline & Characteristics & No & Percentage \\
\hline \multirow{5}{*}{ Burnout } & No Burnout (less than 34) & 25 & 3.1 \\
\hline & Mild Burnout (35-49) & 104 & 13.0 \\
\hline & Moderate Burnout (50-64) & 464 & 58.0 \\
\hline & Severe Burnout (65-79) & 157 & 19.6 \\
\hline & Extreme Burnout (above 80) & 50 & 6.3 \\
\hline \multirow{3}{*}{$\begin{array}{l}\text { Emotional } \\
\text { Exhaustions }\end{array}$} & High (27 0r above) & 159 & 19.9 \\
\hline & Moderate (17-26) & 300 & 37.5 \\
\hline & Low $(0-16)$ & 341 & 42.6 \\
\hline \multirow{3}{*}{$\begin{array}{l}\text { Depersonaliz } \\
\text { ation }\end{array}$} & High (13 or above) & 179 & 22.4 \\
\hline & Moderate (7-12) & 173 & 21.6 \\
\hline & Low (0-6) & 448 & 56.0 \\
\hline \multirow{3}{*}{$\begin{array}{l}\text { Personal } \\
\text { Accomplish } \\
\text { ment }\end{array}$} & High $(0-31)$ & 327 & 40.9 \\
\hline & Moderate (32-38) & 179 & 22.4 \\
\hline & Low (39 or above) & 294 & 36.8 \\
\hline
\end{tabular}

On the other hand, among mild burnout $34.6 \%$ of the cases were aged 18-32 years, among moderate burnout, $35.8 \%$ of the cases aged were aged $33-45$ years, and among severe burnout, $36.9 \%$ were of age $33-45$ years and the remaining $26 \%$ as an extreme category of the same age group, but p-value of 0.20 
Table-II: Association of Burnout with Baseline Parameters

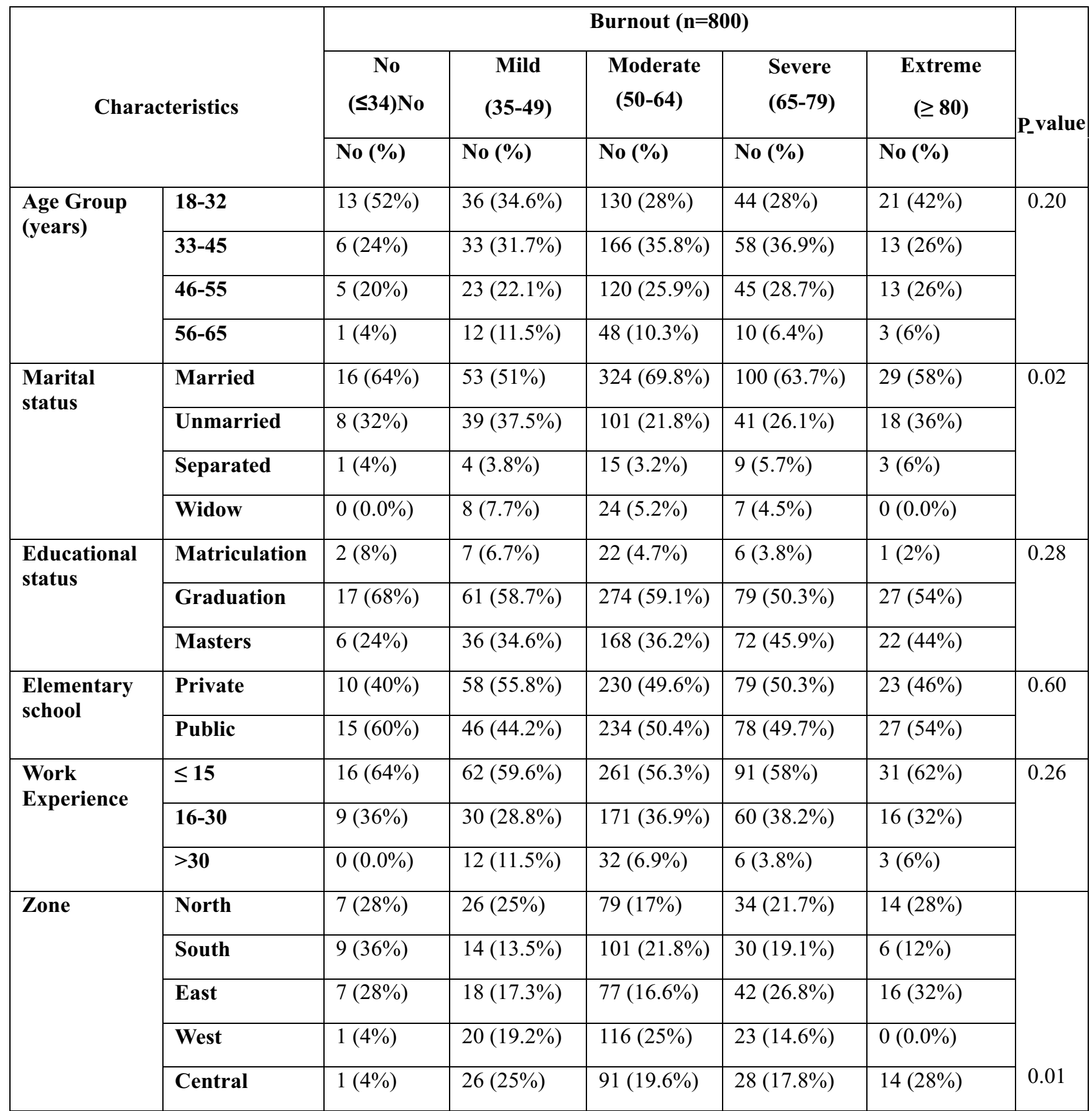

did not give any evidence for association of burnout with age group. (Table-II) In the present study, among mild burnout 51\% were married, and moderate burnout $69.8 \%$ were married, and extreme burnout 58\% were married, p-value of 0.02 showed that burnout gives a significant association with marital status. Furthermore, of those having moderate burnout, 59.1\% were graduates, and those having extreme burnout, $54 \%$ were graduates, but this study didn't give any noteworthy association of burnout with educational status $(\mathrm{p}=0.28)$.
Elementary school and work experience also give an insignificant association with burnout and p-values were observed to be more than 0.05 , however, zone gives significant association, and results showed that $25 \%$ of cases of moderate burnout belong to the west zone, $26.8 \%$ cases of severe burnout belong to east zone and $28 \%$ cases of extreme burnout observed from the central zone.

Table-III gives the mean scores of the MBI scale in marital status and zone, results showed that separated women had higher mean scores on MBI scales compared to married and unmarried women. 
However, these differences were not statistically significant $(\mathrm{p}=0.48)$. Table-III further shows that the East zone respondents have higher MBI scores compared to all other regions, results of the Tukey test showed that the East zone gives significant mean differences for the south and west zone, with a p-value less than 0.05 .

Table-III: Comparison of MBI Scores using One Way ANOVA

\begin{tabular}{|c|c|c|c|c|}
\hline MBI Scores & $\mathbf{N}$ & Mean & $\begin{array}{c}\text { Standard } \\
\text { Deviation }\end{array}$ & p-value \\
\hline \multicolumn{5}{|c|}{ Marital Status } \\
\hline Married & 522 & 58.92 & 11.934 & \multirow{4}{*}{0.48} \\
\hline Unmarried & 207 & 58.63 & 13.627 & \\
\hline Separated & 32 & 61.78 & 14.009 & \\
\hline Widow & 39 & 57.38 & 8.286 & \\
\hline \multicolumn{5}{|l|}{ Zone } \\
\hline North & 160 & 58.23 & 13.971 & \multirow{5}{*}{$0.027^{*}$} \\
\hline South & 160 & 57.73 & 12.019 & \\
\hline East & 160 & $61.51 * *$ & 14.110 & \\
\hline West & 160 & 57.64 & 7.724 & \\
\hline Central & 160 & 59.31 & 12.467 & \\
\hline
\end{tabular}

\section{Discussion}

The purpose of the current study was to find the prevalence of burnout syndrome among female teachers we verified our hypothesis in a similar sample, involving female teachers of elementary schools of Karachi, Pakistan. We also focus onto examine the link between job burnout in relation to public and private sector schools. Furthermore, the study also observed the relationship of burnout with various demographics like age factor, spousal status, literacy rate, teaching practice, and district (zone).

The result revealed that out of 800 female participants working in different elementary schools of Karachi, $96.9 \%$ were found as a case of varying degrees of burnout. The present findings confirmed the three key factors of burnout as evaluated by MBI. It confirms the prevalence of
Burnout among school teachers as proved by previous studies. ${ }^{8}$ Prior research studies, ${ }^{8,19}$ conducted concluded that there was a higher degree of burnout among the public sector schools however our research has not found any significant relationship between the two sectors. This is attributed to the different types of stressors faced by the teachers in each sector. Where factors like difficulty in handling pupils, tense interpersonal relationships, ineffective educational reform, excessively high expectations, and staff cutbacks are common in both sectors. Stressors leading to burnout in the private sector are ruled by the excessively high expectation of themselves, fussy parental dealing, increase time demand, clerical duties, excessive workload, and lack of acknowledgment. Similarly, the stressor of public sectors was found to be overpopulated classrooms, financial constraint, lack of supplies, inadequate discipline policies, lack of leadership and poor school condition. The types of stressors in both sectors are different but the buildup of stress leading to burnout syndrome was a common observation in both two areas. ' Comparison of marital status, the result obtained suggests that the level of burnout varies depending on the marital status of the participants. Studies ${ }^{20,21}$ show a higher percentage of burnout in married participants in comparison with other categories like the results reported by this study.

Comparison with zones, there is a significant association observed between the 5 zones and the level of burnout among the east zone stands out and shows a significant difference from the south and west zone this may be contributed to a change in the work environment and job policies. Demographics of age, educational qualification, and work experience did not show any significant variations in the result obtained this result is parallel to the results obtained by other studies as well. ${ }^{20,21}$ The existing outcomes may have significant suggestions for future research work. Results showed that the prevalence of burnout among teaching faculty is an important issue that may lead to an unhealthy stressful work environment, decreased educational reforms, low morale for students, and decrease success rate of the students.

The present study explains and explores the relationship with different demographics. Knowledge or awareness of the most important stress factors may help in reducing burnout. Study outcomes accentuate the necessity to have a vigilant awareness by the school administration on the 
problem of teacher burnout. An unperturbed and dynamic instructor is more fruitful for the students as compared to a disconsolate one. School administrations must develop new strategies and assign objectives to the teachers with enough resources and rewards to achieve their goals. ${ }^{18}$

The study has some limitations like the statistics was collected from the schools of Karachi, Pakistan, so we cannot compare our findings to other areas of Pakistan as well as gender was not included in it. Prospective motives of teacher burnout must be explored to identify its influences on different teachers and their organizations.

\section{Conclusion}

Our results showed a high prevalence of burnout and the positive association of Burnout with marital status and zones. The policymakers should consider appropriate measures while making decisions regarding school teachers in the public and private sectors.

Authors Contribution: AR: Conception of work, Acquisition and Analysis of Data and drafting. AM: Design of work and revising. PY: Interpretation of data and revising. $\mathbf{A H}$ : Conception of work and revising. AK, AR, MW: Design of work and drafting FA, FZ, AAC: Acquisition and analysis of data and revising. All authors critically revised and approve its final version.

Conflict of Interest: Authors has declared no conflict of interest.

Sources of Funding: The source of funding was self.

\section{Disclaimer: None}

\section{References}

1. Luis moya-Alibiol Masa S. Burnout as an important in the Psychophysiology Responses to a Work Day in Teachers. Stress and Health. 2010;26:382-93.

2. Shahana Naz AH, Aftab Asif. Burnout and quality of life in nurses of a tertiary care hospital in Pakistan. 2016;66:53236.

3. Arnold B. Bakker WBS, Evangelia Demerouti, Peter P.M Jenssen, Renee Van Der Hulst, Janneke Brouwer. Using Equity Theory to Examine the Difference Between Burnout and Depression. Anxiety, Stress \& Coping. 2008;13:247-68.

4. Hyojung Shin HN, Yoojin Jang, Yang Min Park, Sang Min Lee. A longitudinal examination of relationship between teacher burnout and depression. Journal of Employment Counseling. 2013;50:124-37.
5. Lavancho G. Burnout Syndrome and Type A Behavior in Nurses and Teachers in SICILY. Psychological Reports. 1997;81:523-28.

6. Muhammad Masood Khokhar MAC, Nausheen Bakht, Aden Alvi, Madiha Mohyuddin. Burnout among Female Nursing Students. Pak Armed Forces Med Journal. 2016;66(6):87680 .

7. Teacher burnout. Ava i lable from: https://www.enotes.com/research-starters/teacher-burnout.

8. Sewell MHAJ. Stress and Burnout in Rural and Urban Secondary school Teaches. The Journal of Educational Research. 2010.

9. Mary Beth G. Anderson EFI. Teacher Motivation and Its Relationship to Burnout. Educational Administration Quarterly. 1984;20(2):109-32.

10. Yue ZYaY. Causes for Burnout Among Secondary and Elementary School Teachers and Preventive Strategies. Chinese Education and Society 2007;40(5):78-85.

11. Kokkinos CM. Job stressors, personality, and burnout in primary school teachers. British Journal of Educational Psychology. 2007;77:229-43.

12. Ralf Schwarzer T. Teacher Burnout in Hong Kong and Germany: A Cross-Cultural Validation of the Maslach Burnout Inventory. Anxiety, Stress \& Coping 2008;13:30926.

13. Gold Y. The Factorial validity Of The Maslach Burnout Inventory In A Sample Of California Elementary And Junior High School Classroom Teachers. Educational and Psychological Measurement 1984;44:1009-16.

14. Muhammad Ashraf Chaudhry MMK, Muhammad Waseem, Zara Zafar Alvi,, Haq AIu. Prevalence and associated factors of burnout among military Doctors in Pakistan. Pak Armed Forces Med Journal. 2015;65(5):669-73.

15. Yumna Muzafar HHK, Huma Ashraf, Waqas Hussain, Hifsa Sajid, Marium, Tahir AR, et al. Burnout and its Associated Factors in Medical Students of Lahore, Pakistan. Cureus 2015:1-12.

16. Rancer Tas. The Relationship between Trait Verbal Aggressive and Teacher Burnout Syndrome in K-12 Teachers. Communication Research Reports 2008;25; 86-89

17. Martinez AIFaLF. Presenteeism and burnout among teachers in public and private Portuguese elementary schools. The Journal Of Human Resources Management. 2012;23:438090.

18. Jesus Montero-Marin Pk, Ricardo Araya, Margarita Gili, Javier Garcia-Campayo. Towards a brief definition of burnout syndrome by subtypes : Development of the "Burnout Clinical subtype Questionnaire". Health Qual Life Outcomes 2011 Sep 20;9:74. doi: 10.1186/1477-7525-9-74

19. Aristides I. Ferreira, Luis F. Martinez. Presenteeism and burnout among teachers in public and private Portuguese elementary schools, the international journal of human resource management, 2012, 23:20, 4380-4390.

20. Abdul Haque, Mohammad Shakeel Aslam. The influence of demographic on job burnout. far east journal of psychology and burnout. 2011;4(2):57-72.

21. Joachim bauer AS, Kathrina Virnich, Karen Wissing, Udo Muller, Michael Wirsching, Uwe Schaarschmidt. Correlation between burnout syndrome and psychological and psychosomatic symptoms among teachers. Int Arch Occup Environ Health. 2005;79:199-2014. 